\title{
System Architecture Directions for a Software-Defined Lighting Infrastructure
}

\author{
Ye-Sheng Kuo, Pat Pannuto, and Prabal Dutta \\ Electrical Engineering and Computer Science Department \\ University of Michigan, Ann Arbor, Ml 48109 \\ \{samkuo,ppannuto,prabal\}@umich.edu
}

\begin{abstract}
After years of development, cost-effective, energy-efficient, and longlasting solid-state lighting technology is finally a viable alternative to incandescent and fluorescent lights. Unfortunately, the remarkable march of semiconductor technology into the lighting industry is almost entirely in the form of a substitute good-one kind of lighting technology that replaces another-but this, we argue, squanders a unique opportunity for lighting to enable a bevy of new applications. In this paper, we discuss applications in health, energy efficiency, entertainment, communications, indoor positioning, device configuration, and time synchronization. We then prototype several of the indoor applications to explore a software-defined lighting (SDL) architecture that could support them.

Using our prototyped applications, we next take a primitive stab at demonstrating application coexistence, multiplexing multiple applications on a single lighting network. A major question raised by this effort is how to multiplex these various applications in a more principled manner on a shared lighting infrastructure whose primary role is illumination (implying that any human-perceptible flicker or flashing will be unacceptable). Looking ahead, we draw inspiration from software-defined networking's approach to sharing the network, and software-defined radios' approach to processing waveforms, to sketch the beginnings of an SDL architecture and its application programming interfaces.
\end{abstract}

\section{INTRODUCTION}

Indoor lighting is undergoing a renaissance. The staple of illumination for one and a half centuries, the incandescent bulb, is being phased out all around the world $[8,19,26]$. While intermediate technologies such as compact fluorescent bulbs provided the impetus, the emergence of energy-efficient, cost-effective LED lighting is leading to global adoption of the new illumination technology. LED lighting efficiency is over $100 \mathrm{~lm} / \mathrm{W}$, higher than nearly any other technology, and average LED lifetime exceeds 50,000 h, longest among all lighting technologies [3,21]. LED lights offer a compact form factor, high color rendering index (CRI), high performance in low temperature, and a reduction in environmentally dangerous materials such as mercury.

Permission to make digital or hard copies of part or all of this work is granted without fee provided that copies are not made or distributed for profit or commercial advantage and that copies bear this notice and the full citation on the first page.

Copyright is held by the authors.

VLCS'14, September 7, 2014, Maui, Hawaii, USA.

ACM 978-1-4503-3067-1/14/09.

http://dx.doi.org/10.1145/2643164.2643166

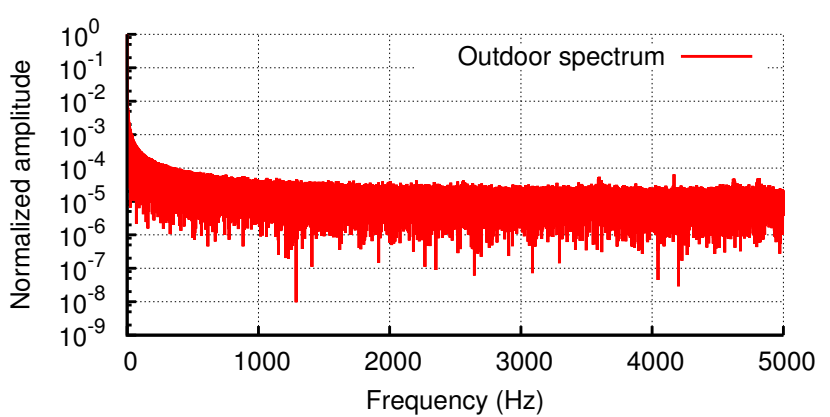

(a) Outdoor spectrum

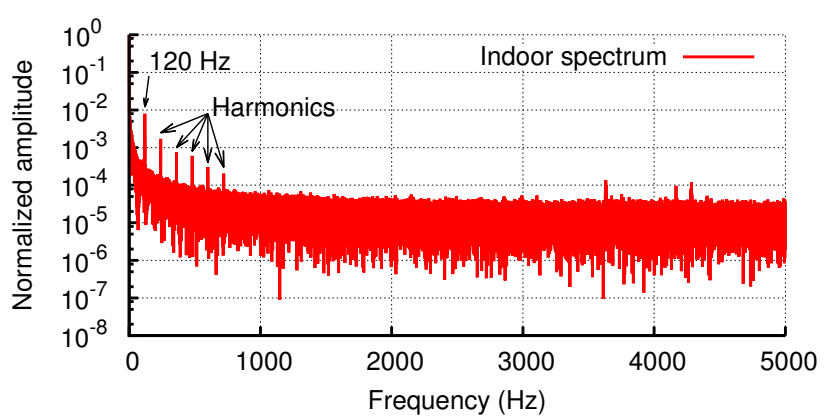

(b) Indoor spectrum

Figure 1: Optical power spectrum of an outdoor environment and a typical fluorescent tube-lit office space. Indoor spectrum is largely unused, with the exception of narrowband spikes at $120 \mathrm{~Hz}$, and their harmonics (due to AC zero crossings that cause the fluorescent light to flicker). Both indoors and outdoors the visible light spectrum is largely unused suggesting it is a rich medium for new communication and other technologies.

One key difference between LED and other lighting technologies is that LEDs are capable of rapidly switching state, much faster than humans can perceive. Hence, high frequency-modulated LED lighting offers a communication channel that is imperceptible to humans. Figure 1 shows the visible light spectrum outdoors and in an office illuminated by a fluorescent light. Indoors, the largest peak occurs at twice the AC mains frequency, $120 \mathrm{~Hz}$, as the fluorescent lights oscillate with each AC zero crossing. More importantly, the harmonic peaks are also narrow band, leaving the majority of the visible spectrum unused. New opportunities are enabled by the largely unused and unregulated spectrum, and the ubiquitous nature of indoor lighting, creating a medium upon which to build applications and services that leverage visible light. 
Many applications are possible with minimal changes to commercial LED luminaires - the addition of a transistor coupled with a microcontroller or digital logic. Other applications require more capable frontends-custom RGBW LED drivers and high-speed optical receivers, for example. This leads us to conclude that a useful exploration of the design space requires an open, experimental, and extensible platform for the luminaires, their optical interfaces, and their communication backhauls. We envision a data plane that consists of a mesh network of luminaires that route IP traffic across their optical, RF, Ethernet, and powerline carrier interfaces, a cloudletmanaged control plane that synchronizes and coordinates nearby luminaires at the physical and link layers, and a programming interface that allows multiple applications to share the lighting infrastructure.

In this paper, we identify an array of applications enabled by visible light communications (VLC) and explore architectural directions for software-defined lighting (SDL) that enable sharing of this medium, are backwards-compatible with existing infrastructure, and are forward-looking with an eye toward supporting new use cases. Our approach is motivated by the recent traction in software-defined networks (SDNs) $[4,9,25,28]$, which open enterprise networks to experimentation, and software-defined radios (SDRs) [20], which blur historical boundaries to make communications more flexible. We propose an initial architecture to explore key questions: how the visible spectrum should be allocated and shared, how applications will access and utilize this new medium, and what workloads can and cannot be supported by these design decisions.

\section{MOTIVATING APPLICATIONS}

As global LED lighting adoption increases, many postillumination lighting applications in health, energy, entertainment, communications, indoor positioning, device configuration, and time synchronization, among many others will emerge. Here, we identify some current opportunities, review research efforts already underway, and speculate on potential future applications.

Health. Light exposure to the eyes is the strongest effector of entrainment, or synchronization, of the internal circadian rhythm with the external environment. The timing, length, intensity, and wavelength of light to which one is exposed all affect entrainment $[2,6]$. We envision a future in which smart badges capture individual exposure parameters, communicate them to the surrounding environment, and securely task nearby lights to adjust spectrum and intensity to compensate for poor exposure, complementing approaches like the University of Michigan-developed Entrain mobile app [12].

Energy Efficiency. Lighting accounts for $26 \%$ of electricity consumption in commercial buildings and $19 \%$ of total electricity use in the U.S [21,27]. Studies have shown that giving occupants fine-grained control over their own lighting can lead to substantial energy savings. Similarly, automated control systems could use mobile phones to detect ambient light levels and provide closed loop feedback to lighting control systems. These control systems could also adjust the lighting level dynamically in response to daylight, saving additional energy. In addition, user location and information profiles can be integrated in control loops to provide human tracking, personalized lighting environments, and energy savings.

Entertainment. Light intensity and color can be controlled by end users, with their mobile phones or programmatically, ${ }^{1}$ to allow people to tailor their environments for fun and whimsy. Taken further, one might imagine lighting that flashes with the ambient music's rhythm and changes color or intensity in response to its spectrogram; future videos could include a "lighting track" akin to current surround sound tracks to enhance viewing experience.

\footnotetext{
${ }^{1}$ http://blinken.eecs.umich.edu
}

\begin{tabular}{|c|c|c|c|c|c|}
\hline & EZ [5] & Radar [1] & Horus [30] & Epsilon [18] & Luxapose [15] \\
\hline Accuracy & $2-7 \mathrm{~m}$ & $3-5 \mathrm{~m}$ & $\sim 1 \mathrm{~m}$ & $\sim 0.4 \mathrm{~m}$ & $\sim 0.1 \mathrm{~m}$ \\
\hline Technology & RF & RF & RF & VLC & VLC \\
\hline Method & Model & FP & FP & Model & AoA \\
\hline Overhead & Minimum & WD & WD & DC & DC \\
\hline
\end{tabular}

Table 1: Comparison of WiFi- and VLC-based localization. FP, WD, AoA, and DC are fingerprinting, war-driving, angle-of-arrival, and device configuration, respectively. Our prior work in VLC-based localization, Luxapose [15], leverages existing smartphones, requires no hardware beyond an SDL deployment, and achieves the best indoor location accuracy to date.

Communications. LED lighting provides an additional communication channel to mobile devices or other dedicated or multiuse receivers. LED lighting can be used in low-rate data broadcasting or simply to provide network connectivity in locations where RF is unavailable. Notifications or alerts can be integrated with security systems to provide visible alarms for toxic gas or insecure windows. Semantic localization-room or region-level accurate indoor localization - is enabled by broadcasting data from lighting luminaires and receiving them with the integrated cameras on mobile phones [24]. This allows one to establish accurate location context without custom hardware or complex environmental modeling. For example, imagine a student pulls out their phone to reserve the conference room in which they are standing, simply by pressing a button that reserves whichever room the phone detects it is currently located in, secured from misuse by location attestation.

Location Attestation. For some applications, like lighting control, proof of presence is important. This ensures, for example, that only people who are physically present can control the lights in a classroom. To enable such scenarios, we propose location attestation as a service available to cloud or mobile applications. A mobile application that wants access to a room's lights would have to first prove its presence in that room by responding to an optical challenge transmitted by the room's lights. This service would take challenge-response authentication into the physical realm.

Beyond Semantic Indoor Positioning. For many years, researchers have been solving the indoor positioning problem using various range-based or range-free proximity techniques. Most proposed solutions focus on RF fingerprinting, proximity, and ranging; however, RF-based techniques suffer from channel variation, limited bandwidth, or intensive calibration and deliver location accuracies measured in meters. Table 1 compares recent work in indoor positioning and finds that VLC techniques can provide better location accuracy than RF-based approaches, down to decimeter accuracy. ${ }^{2}$

Device Configuration. As sensors shrink in size and grow in number, conventional techniques for programming and configuration of devices become impossible. One possibility for programming and configuration is to employ broadcast optical interfaces, rather than electrical ones. For example, the Electric Imp is a system in an SD card form factor that integrates a microcontroller and WiFi networking. The WiFi configuration (SSID and password) is sent to the Electric Imp optically using a dedicated mobile app [7]. For an even smaller example, the Michigan Micro Mote $\left(\mathrm{M}^{3}\right)$ is a modular system with a microcontroller, radio, camera, energy harvester, and sensors in a $1 \mathrm{~mm}^{3}$ form factor. This "smart dust" system is self-sustaining and nearly invisible. Due to the minute form factor, traditional programming methods that rely on physical connections are difficult or impossible at this scale. For this reason, $\mathrm{M}^{3}$ includes an optical frontend for programming [17]. ${ }^{3}$

\footnotetext{
${ }^{2}$ http://eecs.umich.edu/ prabal/videos/AARR+VLC+VIZ.mov

${ }^{3} \mathrm{http} / / /$ youtu.be/OM8WgnhcyOo
} 
Time Synchronization. Highly-stable, pico- and nano-power clocks are beyond the state-of-the-art, so energy constrained millimeter-scale computers currently keep poor time. As a result, communications guard bands dominate the energy budget of lowpower, wireless sensors. SDL offers one possibility to synchronize sensors with a lower power draw. Researchers have demonstrated a sub-nW optical wake-up receiver whose standby power is three orders of magnitude lower than state-of-art RF wake up radios [11] Such optical wake-up receivers can stay on for entire guard bands without depleting batteries, and in some cases even be always-on. Luminaires could be tasked to program/wake-up sensors simultaneously for high-power RF communication, dramatically reducing radio guard time, providing energy-efficient global time synchronization and high-precision coordination of global tasks. ${ }^{4}$

\section{ARCHITECTURAL DIRECTIONS}

Early work captured the initial landscape of possibilities from when VLC was only a nascent technology [14,23]. Since then, there has been extensive work on optimizing the throughput of the VLC channel [16,29], as well as more recent ideas using visible light for applications beyond pure communication [15, 24], and some preliminary work on integrating VLC with other technology, such as powerline communication [13]. As solid-state lighting begins to mature and policy catches up with technology, the time is ripe to crystallize an SDL architecture, while at the same time maintaining sufficient flexibility to support unforeseen directions.

To enable explorations of VLC-enabled networks through research-enabling research, we propose the architecture shown in Figure 2. In this architecture, each luminaire has a local control unit responsible for the visible light output from each node- the data plane - transmitted to a variety of receivers. Actual control of what and when the luminaire emits is delegated to a nearby cloud or cloudlet-the control plane. The VLC component of the architecture is independent of the inter-luminaire backhaul, however, we note that the choice of backhaul will have an impact on the capabilities of the overall SDL system. For example, the connectivity of an Ethernet or powerline-backed network relies on physical network topology, whereas an RF-based approach relies on the geographic distribution of the lights and the nearby environment.

This architecture provides the needed experimental flexibility for the research community but it is grounded in decades of investments in Internet technologies - the luminaires act as IP routers capable of participating in mesh routing protocols and forwarding traffic across their multiple interfaces. This architecture also borrows concepts from software-defined networking, including a controller that can manage flows end-to-end (in this case, between applications and optical or radio frontends), and software-defined radios, for programmatic manipulation of physical layer waveforms. As presented, this architecture focuses on flexibility, seeking to enable the widest class of applications, without imposing undue complexity burdens by leveraging existing infrastructure (IP) and experience (SDN, SDR) in modular design.

\section{DESIGN CONSIDERATIONS}

Although SDL presents many opportunities for new applications, it also raises many new challenges across the system stack. While some of these challenges may be apparent from the outset, many others will not reveal themselves until experimental systems are designed and deployed. We anticipate a number of challenges, as described below, and expect others will emerge as the proposed research unfolds.

\footnotetext{
${ }^{4}$ http://eecs.umich.edu/ prabal/videos/ElectricImp+SDL.mov
}

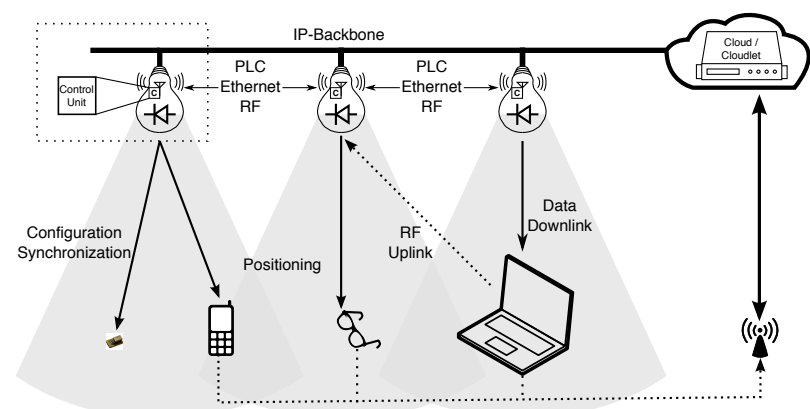

Figure 2: Proposed architecture for software-defined lighting. The architectural elements include: (i) luminaires (the lights themselves), (ii) a Internet Protocol (IP)-based backbone network running over RF, powerline, and/or Ethernet that connects the lights to enterprise IP networks, (iii) cloud or cloudlet servers that manage and control the lights and offload computation for local clients, and (iv) receivers like sensors, smartphones, computational eyeglasses, and cameraequipped laptops that request and/or receive data, computational resources, or other services. This general, flexible, and extensible architecture provides the foundation for a preliminary SDL system, enabling a diverse array of new applications while leveraging wellknown successes and technologies from existing systems.

Cost and Value. For eventual adoption, either the cost of smart lighting must become marginal or its value must become substantial-in the form of new applications enabled because of the lighting system. Practically, this means minor modifications of commercial products will be more palatable to adoption, but significant functional enhancements that enable new revenues streams could also emerge. The challenge lies in determining how to best leverage minimal additional infrastructure to support applications that provide value to the owners or occupants of indoor spaces without inhibiting the exploration of radical ideas. These must be identified, prototyped, and evaluated to make smart, software-defined lighting a commercial reality, and the value of applications must exceed the cost of deploying them.

Flexibility. Balancing long-term cost and value concerns with the flexibility needed for immediate experimentation poses a tradeoff. Specifically, we need a powerful enough platform to test ideas but a clear pathway to inexpensively realizing them. To explore this, we argue for a multi-pronged approach: pushing the extremes in power and performance of solid-state lighting transmitters and receivers, from IEEE 802.15.7 standards compliant technology to nano-watt smart dust receivers with custom circuits and realizations based on slight modifications to existing products to ground exploration.

Networking. Many of the envisioned applications for softwaredefined lighting require dynamic information to be received (and subsequently transmitted) by the lighting infrastructure. How should this data be transmitted to the lights in the first place? Many options exist, including Ethernet, PLC, and RF, but they occupy different points in the space of cost, complexity, and data rate. Both Ethernet and PLC provide networking and power delivery, reducing wire counts. However, many existing buildings do not have Ethernet outlets everywhere, requiring costly infrastructure change to support networking. On the other hand, PLC provides lower data rates than Ethernet and network connectivity depends on the layout of the power distribution network. Commercial buildings often have many electrically distinct domains, separated by transformers that isolate PLC signals. Isolated PLC nodes may require RF links, or Ethernet/PoE support, to form complete networks. This suggests that a multihop, multi-link protocol like IP may be ideal. 


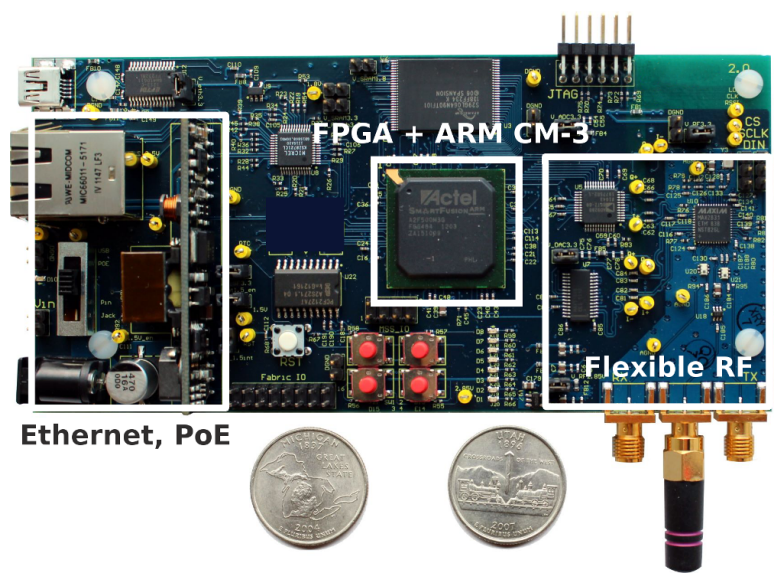

Figure 3: Prototype platform. This platform integrates Ethernet, PoE, a flexible RF SDR frontend that supports 802.15.4, and a digital control interface to the optical RX and TX frontends. The node runs uClinux on an ARM Cortex-M3.

Synchronization. In some cases (e.g. with cameras as line-ofsight receivers), it is possible for multiple lights to transmit data through optical modulation since each pixel in the image plane corresponds to a different region in the world view. In other cases, diffusing receivers (e.g. photodiodes) will mix incoming light from multiple sources, so either the transmissions must be time synchronized or they must employ some form of orthogonality, like TDMA or FDMA. This creates a space of tradeoffs in protocol complexity, computing requirements, and communications efficiency.

Modulation. For applications that require lights to transmit information optically, how should the data be encoded and modulated? The answer depends in part on the receiver, in part on the illumination, and in part on any applicable standards. Although VLC standards like IEEE 802.15.7 exist, they make many assumptions about the available infrastructure and assume control over receivers. This makes adoption challenging as it requires two different industries-lighting and consumer electronics-to agree to absorb costs without any clearly-defined market opportunity. A different approach, which we advocate, makes physical layer modulation schemes programmable. Today, we can leverage existing and emerging receivers, like smartphones and smart dust, respectively, and tailor visible light communications to each, exploring opportunities before market forces conspire to make them profitable. Tomorrow, we can decide which modulations schemes are best given the computational, communications, and energy resources available for transmitters and receivers.

Multiplexing. A central requirement of reusing the lighting infrastructure for other applications is that these applications must share the lights, through some kind of multiplexing, to support the various applications without compromising on the lighting's primary role in illumination. The questions are not limited to the physical layer, however. For example, in applications that require spatial control over lights by users, how should different principals (applications, services, or users) control the lights and resolve conflicts?

Backward-Compatibility. Software-defined lighting must offer a modicum of compatibility with existing lighting systems, while new functions should support incremental deployment rather than require wholesale infrastructure replacement. Practically, this means that preference should be given to platforms that can replace Edison, can, or fluorescent lamps for immediate adoption, ensuring that these platforms can be used both for immediate lighting and alternate uses.

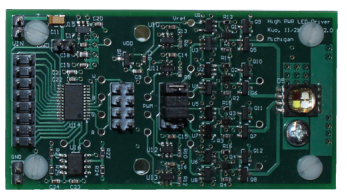

(a) RGBW VLC TX

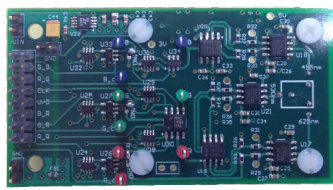

(d) RGB VLC linear RX

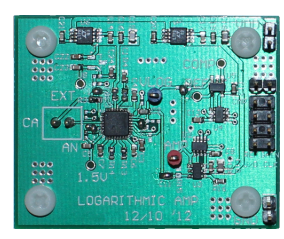

(b) VLC $\log \mathrm{RX}$

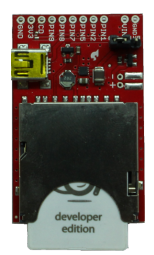

(c) Electric Imp

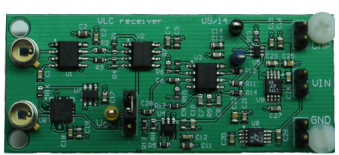

(e) Dual VLC RX

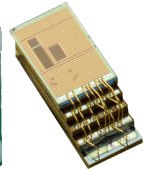

(f) $\mathrm{M}^{3}$
Figure 4: VLC Transceivers. VLC receivers must choose between (d) bandwidth, (b) dynamic range, or (e) the cost of including both. SDL may not always control the receivers, as in the case of (c) commercial or (f) research devices.

\section{PRELIMINARY EXPERIENCES}

To explore the SDL design space, we prototype an array of transmitters and receivers in support of some of the envisioned applications. To explore their interoperability and the interoperability of various protocols on an SDL network, we explore simple multiplexing techniques in a proto-SDL deployment in a lab-scale setting.

\subsection{Full-Flexibility Luminaires}

For a full-flexibility luminaire controller, we prototype the platform shown in Figure 3 which features reconfigurable wireless interfaces in the $2.4 \mathrm{GHz}$ ISM band, supports Ethernet backbone connectivity, and leverages Power-over-Ethernet $(\mathrm{PoE})$ for combined power and data. This controller integrates both a SDR and a SDL controller, including a digital control interface to optical transmitter and receiver frontends. This design, integrating SDL into an SDR platform, is a recognition of the many similarities between RF and visible light modulation, and also the potential benefits that could be enabled by the co-design of RF and visible light protocols.

Much like an SDR's capabilities depend heavily on its RF frontend, a VLC transmitter's capabilities depend heavily on the driver circuitry and LED frontend. We design a dedicated RGBW VLC TX frontend for our hybrid SDR/SDL platform, seen in Figure 4a, that is capable of high performance $(>20 \mathrm{MHz}$ ) switching on three distinct optical channels — red, green, and blue-as well as white, allowing partial 802.15.7 support for on-off keying and variable pulse position modulation. The rest of Figure 4 explores various transceiver design points, both custom and commercial.

\subsection{Cost-Effective Luminaires}

While our full-flexibility controller and transmitter-capable of generating and driving complex waveforms-provide many options, they are also costly. To address this, we explore the viability of modifying commercial off-the-shelf (COTS) LED lights to support SDL and the capability of more fixed-function controllers. As shown in Figure 5, we modify the power supplies of nearly thirty Commercial Electric T60, T65, T66, and T67, as well as several Utilitech Pro, lights in only a few hours. From each modification, we expose an open circuit that when closed activates the light. We are able to interface our full-flexibility controller to drive the modified COTS lights, however they are unsurprisingly less responsive (order $\sim 1 \mathrm{MHz}$ ) as their driver circuits are not optimized for rapid cycling. 


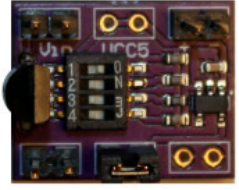

(a) Oscillator

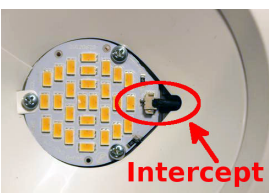

(b) LED Modifications

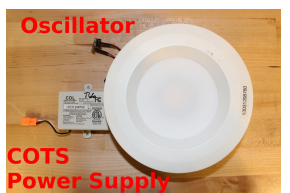

(c) Complete Package.
Figure 5: COTS Modifications. With only trivial modifications, we can inject control signals into existing COTS LEDs. This modification exposes an on-off control line, that can be controlled by our full-flexibility controller or by a simpler controller, such as the oscillator shown in (a).

In lieu of the costly full-flexibility controller, we also investigate the viability of a lower-cost, fixed function, digital controller. Our Luxapose system for VLC indoor positioning requires only that each luminaire emit a square wave at a unique frequency [15]. We build a controller with a simple voltage controlled oscillator as a controller for this application. As seen in Figure 5a, we include a DIP switch for local control of the frequency, but also add pins to allow an external controller to take over the luminaire, preserving potential future flexibility.

\subsection{Multiplexing VLC Applications}

Various VLC applications and receivers impose conflicting requirements. Luxapose leverages a smartphone camera, whose many pixels are capable of perceiving different transmitters. This is key to the positioning primitive, which requires that each transmitter is emitting a different frequency. The $\mathrm{M}^{3}$ node, on the other hand, has a diffusing receiver-a single photodiode. To recover a meaningful signal, the $\mathrm{M}^{3}$ requires that all transmitters in its field of view send the same signal with tight synchronization to avoid interference.

In Figure 6 we show a simple TDMA [22] approach to supporting these two applications using the same lighting infrastructure. We leverage the SDR aspects of our full-flexibility controller and a recent low-power MAC protocol, Glossy [10], to achieve the tight timing synchronization required to send the synchronization commands to the $\mathrm{M}^{3}$ chips. Figure 7 shows the range of components in our proto-SDL implementation-transmitters, controllers, and receivers-that we are integrating to realize SDL.

\section{CONCLUSIONS}

Software-defined lighting is an emerging technology that is unconstrained by legacy considerations, heavy spectrum usage, and the regulation and restrictions imposed on most other communication mediums. A burgeoning VLC research field has primed SDL with a diverse array of communications methods, while the architectural lessons from SDRs and SDNs inform the hardware, firmware, software, and system design in a way that is typically unavailable to nascent technologies. Leveraging this prior work in VLC, SDR, and SDN, this paper offers one vision for an SDL architecture, and some initial experiences realizing it. While we find some success in siloed applications, and our initial attempts at integrating them, we are far from having identified the key elements of the SDL control and data planes, and the myriad components that will constitute them. Looking ahead, with hardware in hand, the key questions that must be addressed include how to share the infrastructure across many applications (including lighting), how to expose programmatic control of the system's many elements to applications, and how to route the right data to the right place at precisely the right time.
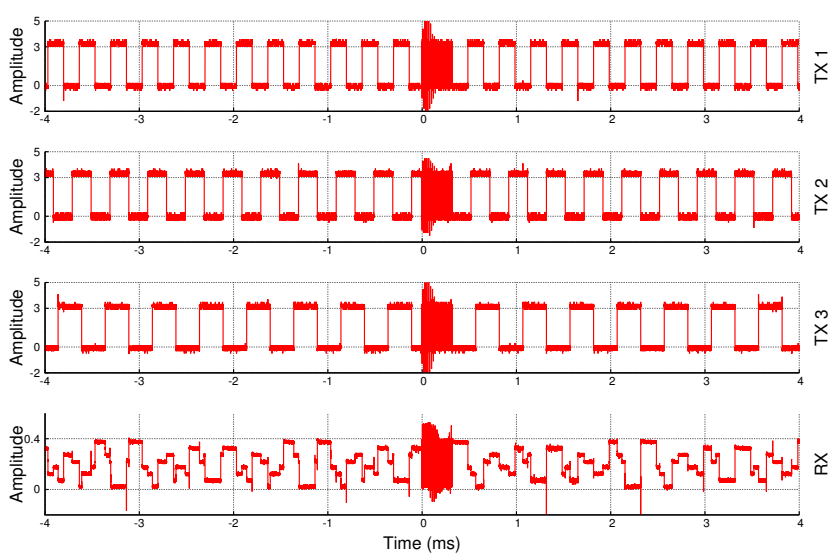

(a) Localization ( -4 to $0 \mathrm{~ms}, 0.3$ to $4 \mathrm{~ms}$ ) and synchronization ( 0 to $0.3 \mathrm{~ms}$ ).
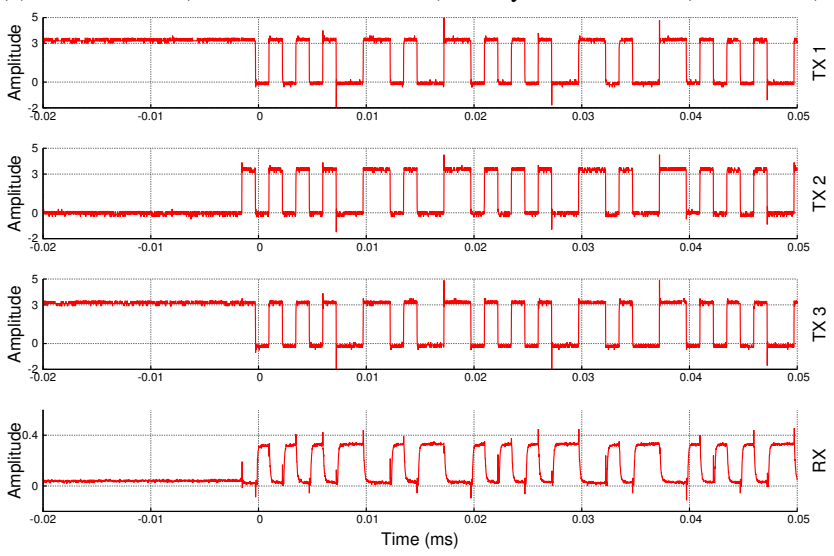

(b) Detail view of a synchronization sequence.

Figure 6: Multiplexing services over a VLC link.

(a) Three transmitters (top three rows) provide both localization and synchronization services. Localization requires the transmitters to transmit unique idle patterns (three different frequencies) to CMOS camera imagers while synchronization requires them to transmit identical data with symbol-level synchronization to photodiodes. (b) A zoomed-in view of synchronization transmission from the same three transmitters and as captured by a diffusing receiver. Tightly synchronized transmission allows diffusion-based receivers like photodiodes to correctly receive data without inter-symbol interference from adjacent transmitters.

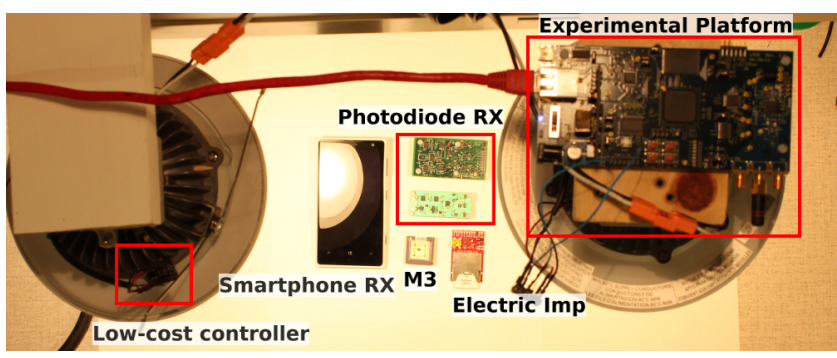

Figure 7: SDL elements. Two commercial LED lights are controlled by two controller prototypes, a high-cost experimental platform and a low-cost fixed-function controller respectively. The lighting data are being received by several possible receivers including photodiode receivers- $\mathrm{M}^{3}$ "smart dust" [17] and an Electric Imp [7]—and a CMOS array inside of a smartphone. 


\section{ACKNOWLEDGMENTS}

This work was supported in part by the TerraSwarm Research Center, one of six centers supported by the STARnet phase of the Focus Center Research Program (FCRP), a Semiconductor Research Corporation program sponsored by MARCO and DARPA. This research was conducted with Government support under and awarded by DoD, Air Force Office of Scientific Research, National Defense Science and Engineering Graduate (NDSEG) Fellowship, 32 CFR 168a. This material is based upon work partially supported by the National Science Foundation under grants CNS-0964120, CNS1111541, and CNS-1350967, and generous gifts from Intel, Qualcomm, and Texas Instruments.

\section{REFERENCES}

[1] P. Bahl and V. N. Padmanabhan. RADAR: An in-building RF-based user location and tracking system. In Proc. 19th Annual Joint Conference of the IEEE Computer and Communications Societies. (INFOCOM 'O0), volume 2, pages 775-784, 2000.

[2] D. B. Boivin, J. F. Duffy, R. E. Kronauer, and C. A. Czeisler. Dose-response relationships for resetting of human circadian clock by light. 1996.

[3] C. Branas, F. Azcondo, and J. Alonso. Solid-State Lighting: A System Review. IEEE Industrial Electronics Magazine, 7(4):6-14, 2013.

[4] M. Casado, M. J. Freedman, J. Pettit, J. Luo, N. McKeown, and S. Shenker. Ethane: Taking control of the enterprise. In Proc. of the 2007 Conference on Applications, Technologies, Architectures, and Protocols for Computer Communications (SIGCOMM '07), pages 1-12. ACM, 2007.

[5] K. Chintalapudi, A. Padmanabha Iyer, and V. N. Padmanabhan. Indoor localization without the pain. In Proc. of the 16th ACM Annual International Conference on Mobile Computing and Networking (MobiCom '10), 2010.

[6] C. A. Czeisler. The effect of light on the human circadian pacemaker. Circadian clocks and their adjustment, 254:302, 1995.

[7] Electric Imp. Electric Imp datasheet. https://electricimp.com/docs/attachments/hardware/ datasheets/imp001_specification.pdf.

[8] European Commission. Commission adopts two regulations to progressively remove from the market non-efficient light bulbs. http://europa.eu/rapid/press-release_IP-09-411_en.pdf, 2009.

[9] N. Feamster, J. Rexford, and E. Zegura. The road to SDN. Quеие, 11(12):20:20-20:40, 2013.

[10] F. Ferrari, M. Zimmerling, L. Thiele, and O. Saukh. Efficient network flooding and time synchronization with glossy. In Proc. of the 10th International Conference on Information Processing in Sensor Networks (IPSN '11), pages 73-84. IEEE, 2011.

[11] G. Kim, Y. Lee, S. Bang, I. Lee, Y. Kim, D. Sylvester, and D. Blaauw. A $695 \mathrm{pW}$ standby power optical wake-up receiver for wireless sensor nodes. In Custom Integrated Circuits Conference (CICC '12), pages 1-4. IEEE, 2012.

[12] D. F. Kirill Serkh, Olivia Walch. Entrain. http://entrain.math.lsa.umich.edu/.

[13] T. Komine and M. Nakagawa. Integrated system of white LED visible-light communication and power-line communication. Consumer Electronics, IEEE Transactions on, 49(1):71-79, Feb 2003.

[14] T. Komine and M. Nakagawa. Fundamental analysis for visible-light communication system using LED lights.
Consumer Electronics, IEEE Transactions on, 50(1):100-107, Feb 2004.

[15] Y.-S. Kuo, P. Pannuto, K.-J. Hsiao, and P. Dutta. Luxapose: Indoor positioning with mobile phones and visible light. In Proc. of the 19th Annual International Conference on Mobile Computing \& Networking (MobiCom '14), 2014.

[16] H. Le-Minh, D. O’brien, G. Faulkner, L. Zeng, K. Lee, D. Jung, Y. Oh, and E. T. Won. 100-Mb/s NRZ visible light communications using a postequalized white LED. Photonics Technology Letters, IEEE, 21(15):1063-1065, Aug 2009.

[17] Y. Lee, S. Bang, I. Lee, Y. Kim, G. Kim, M. H. Ghaed, P. Pannuto, P. Dutta, D. Sylvester, and D. Blaauw. A modular $1 \mathrm{~mm}^{3}$ die-stacked sensing platform with low power $\mathrm{I}^{2} \mathrm{C}$ inter-die communication and multi-modal energy harvesting. IEEE Journal of Solid-State Circuits, 48(1):229-243, 2013.

[18] L. Li, P. Hu, C. Peng, G. Shen, and F. Zhao. Epsilon: A visible light based positioning system. In Proc. of the 11th USENIX Symposium on Networked Systems Design and Implementation (NSDI '14), pages 331-344, 2014.

[19] Ministerial Council for Energy. Energy Labelling and MEPS Program Regulatory Ruling. Australian Government Department of Industry. http://www.innovation.gov.au/ Energy/EnergyEfficiency/Documents/energy-efficiency/ Regulatory-Ruling-Incandescent-Final-v1.pdf.

[20] J. Mitola. The software radio architecture. IEEE Communications Magazine, 33(5):26-38, 1995.

[21] Navigant Consulting. 2010 U.S. Lighting Market Characterization. 2012.

[22] R. Nelson and L. Kleinrock. Spatial TDMA: A collision-free multihop channel access protocol. IEEE Transactions on Communications, 33(9):934-944, 1985.

[23] D. O’brien, L. Zeng, H. Le-Minh, G. Faulkner, J. Walewski, and S. Randel. Visible light communications: Challenges and possibilities. In Personal, Indoor and Mobile Radio Communications, 2008. PIMRC 2008. IEEE 19th International Symposium on, pages 1-5, Sept 2008.

[24] N. Rajagopal, P. Lazik, and A. Rowe. Visual light landmarks for mobile devices. In Proc. of the 13th ACM/IEEE International Conference on Information Processing in Sensor Networks (IPSN'14), pages 249-260, 2014.

[25] S. Sezer, S. Scott-Hayward, P. Chouhan, B. Fraser, D. Lake, J. Finnegan, N. Viljoen, M. Miller, and N. Rao. Are we ready for SDN? Implementation challenges for software-defined networks. IEEE Communications Magazine, 51(7):36-43, 2013.

[26] The 100th Congress. Energy Independence and Security Act of 2007. Public Law 110-140. U.S. Government Printing Office, 2007. http://www.gpo.gov/fdsys/pkg/ PLAW-110publ140/html/PLAW-110publ140.htm.

[27] U.S. Department of Energy. 2010 Commercial energy end-use splits, by fuel type. Building Energy Data Book, 2012.

[28] J. Van der Merwe, S. Rooney, I. Leslie, and S. Crosby. The Tempest-A practical framework for network programmability. IEEE Network, 12(3):20-28, 1998.

[29] J. Vučić, C. Kottke, S. Nerreter, K.-D. Langer, and J. W. Walewski. $513 \mathrm{mbit} / \mathrm{s}$ visible light communications link based on DMT-modulation of a white LED. J. Lightwave Technol., 28(24):3512-3518, Dec 2010.

[30] M. A. Youssef and A. Agrawala. The Horus WLAN location determination system. In Proc. of the 3rd International Conference on Mobile Systems, Applications, and Services (MobiSys '05), pages 205-218, 2005. 